\title{
Pedestrian Evacuation Modelling with Dynamics Congestion Avoidance
}

\author{
Zongzhi Wang', Tao Chen ${ }^{2}$ \\ ${ }^{1,2}$ Institution of Public Safety Research \\ Tsinghua University, Beijing, China \\ zongzhiwang@hotmail.com; chentao.a@tsinghua.edu.cn
}

\begin{abstract}
With the development of computer technology, pedestrian simulation becomes an efficient method to analyse evacuation efficiency under various scenarios. Some important and common behaviour of pedestrians, congestion detection and avoidance, which is seldom considered in pedestrian simulation complicatedly, are discussed in this paper. A modified cellular automata model considering dynamic congestion detection and avoidance is proposed and applied to simulate two different scenarios to demonstrate the effect of congestion avoidance behaviour, which have a significant improvement on evacuation efficiency. The accuracy and efficacy of this model is verified through the comparison result which is conducted through commercial software, Pathfinder. The modified model shows that with the consideration of congestion avoidance behaviour properly, the evacuation efficiency is improved approximately $40 \%$ than the model proposed by this paper, without the consideration of congestion avoidance behaviour.
\end{abstract}

Keywords: Pedestrian dynamics, Pedestrian simulation, Emergency evacuation, Cellular automata, Congestion avoidance

\section{Introduction}

Pedestrian evacuation simulation is an efficient method to analyse emergency evacuation plan. The intrinsic requirement to improve the accuracy of simulation is to establish movement rules that reflect actual pedestrian behaviour. The impediments to high evacuation efficiency in an evacuation mainly are some 'congestion points', for example, exit doors or narrow corridors. Previous research was conducted in this domain and some inspiring methods were introduced.

While analysing information transmission toward crowds, a social force model considering exit density of pedestrian was built, simulating a four exits square room (Han, Liu. 2017 ${ }^{[1]}$ ). A modification of an existing cellular model was proposed with experiments conducted in a classroom with obstacles (Liu, et al. $2009^{[2]}$ ), taking into consideration of the influence of occupant density around exits. Cellular automata model was employed and modified by researchers to simulate pedestrians' velocity change with different neighbourhood density (Pereira, et al. 2013 ${ }^{[3]}$ ). Research leading by the same researcher (Pereira, et al. $\left.2017^{[4]}\right)$ modified his cellular automata model with introduction of adjust field, the value of which is relevant to congestion nearby and the willingness of congestion avoidance is determined by the weight of adjust field.

More researchers have conducted relevant research on congestion avoidance behaviour, including analysis on the relationship between crowd density and movement velocity (Fang, et al. $2003^{[5]}$ ), modelling the response of behaviour on the presence of other pedestrian (Antonini, et al. 2006 $6^{66}$ ), application of Voronoi diagram to calculate the detour direction (Yunchao, Qu. 2018 ${ }^{[7]}$ ), and the consideration of panic mentality under congestion situation (Jinhuan, Wang. 2015 ${ }^{[8]}$ ).

\section{The Principles of the modified cellular automata model}


Cellular automata model is a discrete model used to simulated pedestrian movement widely. Evaluation area is divided into lattices or cells while pedestrians are viewed particles occupied a single cell at a time, who follow movement rules, in the form of moving probabilities to transfer to neighbour cells.

\subsection{Movement Probability Equations}

Movement probability equations are the most important part of a cellular automata model, of which the equations provide the description of certain human behaviour. Mainly based on Pereira and other researchers' model, the movement probability of pedestrian is presented as following equation.

$$
p_{i j}=N \xi_{i j} \exp \left(K_{s} S_{i j}\right) \exp \left(K_{d} D_{i j}\right) \exp \left(\delta_{a} K_{a} A_{i j}\right)
$$

Where, $N$ is the normalization factor; $\xi_{i j}$ denotes the factor which determines whether the cell $\{\mathrm{i}, \mathrm{j}\}$ is available for a pedestrian; $S_{i j}$ denote the value of static field, which measures the prior movement preference of a pedestrian; $K_{s}$ denotes the sensitive parameter of static field; $D_{i j}$ denotes the value of dynamic field; $K_{d}$ denotes the sensitive parameter of dynamic field; $A_{i j}$ denotes the value of adjust field, which measures the movement preference when the primary exit is jammed; $K_{a}$ denote the sensitive parameter of adjust field; $\delta_{a}$ is an indicator of congestion situation, of which the calculation method is discussed in the next section.

\subsection{Congestion Detection and Avoidance Method}

The imitation of congestion detection and avoidance behaviour of pedestrian is accomplished by the introduction of a quasi-ant colony algorithm combined with the adjust field. Mentioned in Eqs. (1) and previous section, $\delta_{a}$ is an binary variable that submit to some rules to indicate a congestion being detected. . $\varphi$, representing "pheromone", is calculated as Eqs. (2) shown.

$\varphi_{t}=\lambda \times \varphi_{t-1}+x$

Where, $\lambda$ denotes the decay parameter of pheromone; $x$ denotes the number of people in detection area. $\delta_{a}$ follows these rules:

$\delta_{a}=\left\{\begin{array}{l}0 \rightarrow 1, \varphi_{t}>\text { threshold }_{h} \\ 1 \rightarrow 0, \varphi_{t}<\text { threshold }_{l}\end{array}\right.$

The value of threshold $_{h}$ is always higher than the value of threshold $l$ indicating pedestrians' cognition are always lag behind actual information.

\section{Simulations and Results}

Based on Eqs. (1) - (3), two scenarios are established to test the validity and availability of the model, and one of the strengths of the proposed model is tested as well, that due to the introduction of 'pheromone' and $\delta_{a}$, the congestion detection indicator, complex scenario which include more than one congestion point, can be analysed easily.

\subsection{Illustration of Scenarios}

Two artificial scenarios are designed and developed through cellular automata model, and the schematic illustrations of them as well as alternative routes are shown in Fig.1. 

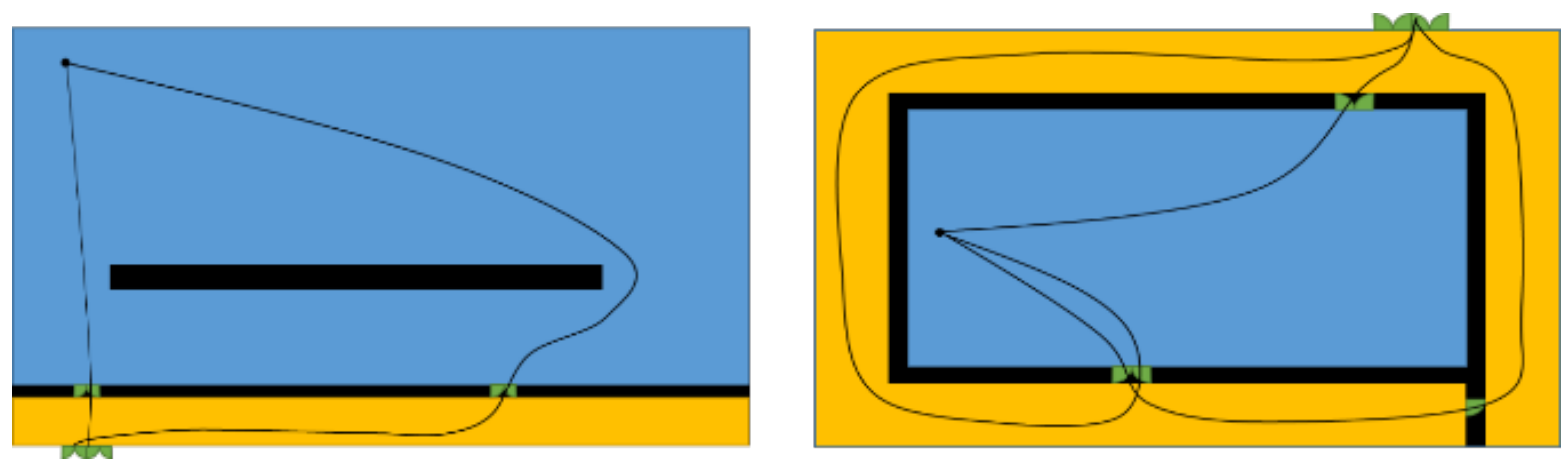

Fig.1: The schematic illustrations of the artificial scenarios

\subsection{Verification of the modified model}

The accuracy of the model is testified through the comparison of the result with the simulation output or commercial software Pathfinder on the less complicate scenario in which the evacuation area is rebuilt with the detour exit sealed, which represents the least complex condition for illustration convenience. The result of validation is shown in Fig.2.

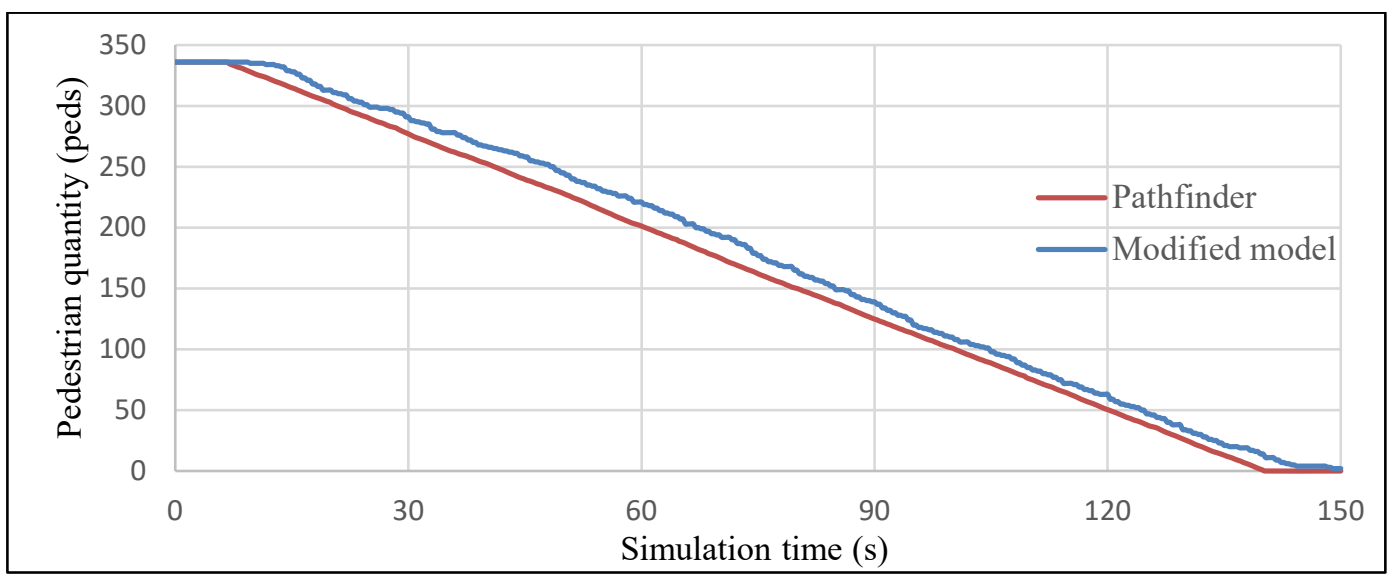

Fig.2: The results of modified model and Pathfinder on same scenario without congestion avoidance behaviour

After the adjustment of the parameters of modified model, the evacuation efficiency error between each result is within the range of $5 \%$ or 15 pedestrians.

\subsection{Brief illustrations of simulations}

The simulation result is ideal that significant improvement on evacuation efficiency are displayed in both scenarios with the introducing of adjust field. Part of the results are shown in Fig.3. The first row images show the evacuation process without the consideration of congestion avoidance behaviour, and therefore, the sensitive parameter of adjust field is set as 0 . The second row images show the imitation of congestion avoidance behaviour, and the evacuation efficient is improved significantly if pedestrians make a detour to avoid congested exit or corridor. Pedestrians detect congestion situations and make decisions dynamically, and thus they may alter their routes according to the situation promptly. 


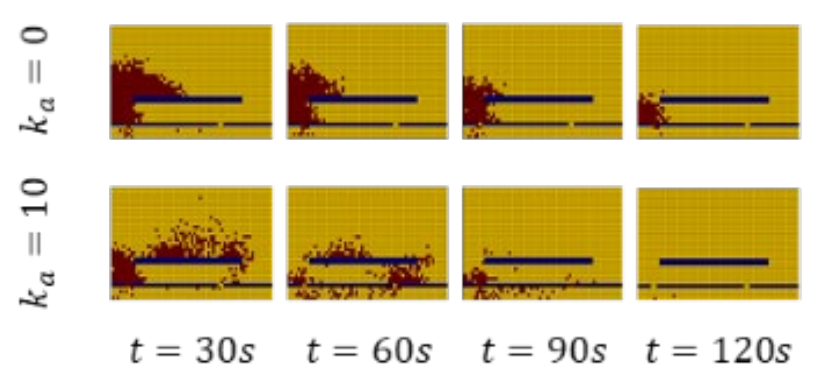

a) Results of less complicated scenario

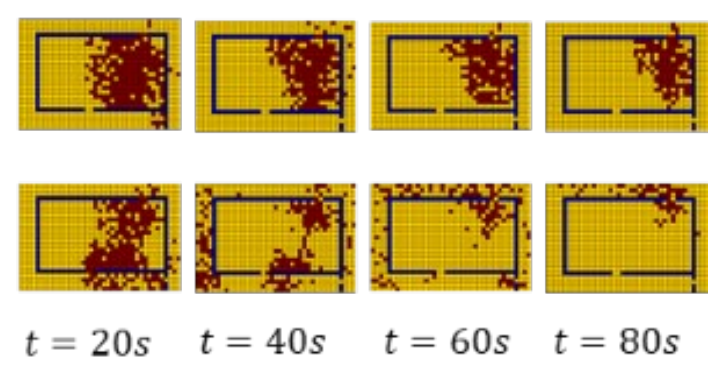

b) Results of complicated scenario

Fig.3: The results of simulation of modified CA model

\section{Conclusion}

Emergency evacuation which takes place in human intensive facility faces impediments of congestion problems. In an ideal condition, pedestrians would make decisions on whether choosing the nearest evacuation route, or moving around when the nearest route is congested. This paper proposed a modified cellular automata model to simulate congestion avoidance behaviour of pedestrians. With the introduction of quasi-ant colony algorithm and adjust field in cellular automata model, the modified model is capable of imitation congestion detection and avoidance behaviour dynamically, while the analysis of complex scenarios can be conducted easily. In two examples proposed in previous section, this model output ideal results that the congestion avoidance behaviour is imitated properly while the evacuation efficiency is improved at $35 \%$ or $43 \%$ maximum, respectively, then the model without congestion avoidance behaviour.

\section{Acknowledgements}

The authors appreciate the projects 71373139 and 71673163 supported by NSFC of China.

\section{References}

[1] Y. Han, and H. Liu, "Modified social force model based on information transmission toward crowd evacuation simulation," Physica A: Statistical Mechanics and its Applications, vol. 469, pp. 499-509, 2017.

[2] S. Liu, L. Yang, T. Fang, and J. Li, "Evacuation from a classroom considering the occupant density around exits," Physica A: Statistical Mechanics and its Applications, vol. 388(9), pp. 1921-1928, 2009.

[3] L. A. Pereira, L. H. Duczmal, and F.R.B. Cruz, "Congested emergency evacuation of a population using a finite automata approach," Safety Science, vol. 51, pp: 267-272, 2013.

[4] L. A. Pereira, D. Burgarelli, L. H. Duczmal, and F.R.B. Cruz, "Emergency evacuation models based on cellular automata with route changes and group fields," Physica A: Statistical Mechanics and its Applications, vol. 473, pp: 97-110, 2017

[5] Fang, Z., S. M. Lo, and J. A. Lu. "On the relationship between crowd density and movement velocity." Fire Safety Journal vol. 38, no.3, pp: 271-283, 2003.

[6] G. Antonini, M. Bierlaire, and M. Weber, "Discrete choice models of pedestrian walking behavior." Transportation Research Part B: Methodological vol. 40, no.8, pp: 667-687, 2006.

[7] Y. Qu, Y. Xiao, J. Wu, T. Tang, and Z. Gao, "Modeling detour behavior of pedestrian dynamics under different conditions." Physica A: Statistical Mechanics and its Applications vol. 492, pp: 1153-1167, 2018.

[8] J. Wang, L. Zhang, Q. Shi, P. Yang, and X. Hu, "Modeling and simulating for congestion pedestrian evacuation with panic." Physica A: Statistical Mechanics and Its Applications vol. 428, pp: 396-409, 2015. 